\title{
Kirchhoff imaging beyond aliasing
}

\author{
Biondo L. Biondi ${ }^{1}$
}

keywords: aliasing, resolution, 3-D migration

\begin{abstract}
I present a method for anti-aliasing Kirchhoff imaging operators that improves the resolution of the image by properly imaging aliased components of the recorded data that would be suppressed by standard anti-aliasing methods for Kirchhoff operators. The proposed method succeeds in imaging "beyond aliasing" without generating aliasing noise because it exploits a priori knowledge on the dipspectrum of the data. Therefore, the proposed method is not of general applicability but it successfully improves the image resolution when a priori assumptions on the dip-spectrum of the data are realistic. The imaging of a salt-dome flanks in the Gulf of Mexico has been enhanced by the application of the proposed method.
\end{abstract}

\section{INTRODUCTION}

High-resolution is a paramount goal of seismic imaging. Unfortunately, we often face a trade-off between resolution and signal-to-noise because of incomplete spatial sampling of the data. To sample the data adequately is expensive, and in many cases outright impossible. Too often the data are not adequately sampled to be imaged with maximum resolution by standard imaging methods. In order to avoid aliasing noise, standard imaging methods discard some of the high-frequency components of the signal that have been recorded. In this process valuable image resolution is lost.

Imaging beyond aliasing is therefore a very active area of research. A promising direction aims to exploit the redundancy of multi-offset data sets to generate aliasfree and high-resolution stacks (Brink et al., 1997) or partial stacks (Chemingui and Biondi, 1998). However, these methods are based on the inversion of prestack imaging operators (DMO or AMO) that are computationally demanding. Another approach based on inversion, but less computationally demanding, is the interpolation of aliased data by prediction-error filters (Crawley, 1998). Wisecup (1998) presented an interesting method to exploit data redundancy over offset to overcome temporal aliasing. The application of his idea to overcome spatial aliasing certainly has potential, but

\footnotetext{
${ }^{1}$ email: biondo@sep.stanford.edu
} 
has not been developed yet. Huygen-migration methods are also promising (Neidell, 1997), although their theoretical development is still not mature.

The goal of the anti-aliasing method presented in this paper is to improve the image resolution by properly imaging some of the aliased components of the recorded data, that would not be imaged by applying standard anti-aliasing methods of Kirchhoff operators (Gray, 1992; Bevc and Claerbout, 1992; Lumley et al., 1994; Abma, 1998). Ours is thus a more modest goal than the ones of the research discussed above. But the proposed method has the advantage of not requiring complex, and sometime unreliable, inversion techniques. In addition, it does not require data redundancy, and thus can be applied to the imaging of minimal data sets (Padhi and Holley, 1997), such as zero-offset data. I show that by a simple modification of the anti-aliasing conditions of the Kirchhoff migration operator, some of the aliased components in the data are nicely imaged, without generating aliasing noise. The proposed "imaging beyond aliasing" is possible by exploiting a priori assumptions on the dip bandwidth of the data. These assumptions are realistic in many important cases, such as the example that I show of the imaging of a the steep flanks of a salt dome in the Gulf of Mexico. Pica (1996) presented a model-based anti-aliasing method that is related to the one presented in this paper. However, his analysis is based on a priori information on the image dips instead of data dips, and it yields different anti-aliasing conditions.

To derive the proposed anti-aliasing method, I analyze in detail the aliasing of Kirchhoff imaging operators. I distinguish the image-space aliasing from the dataspace aliasing, often referred to as operator aliasing. Image aliasing occurs when the imaging operator, represented by its spreading formulation (e.g. ellipsoid for migration) is too coarsely sampled in image space. Dually, operator aliasing occurs when the gathering formulation of the imaging operator (e.g. hyperboloid for migration) is too sparsely sampled. I show that image aliasing is closely related to operator aliasing when the data sampling and the image sampling are the same and the data are not aliased. Therefore, the two concepts are seldom distinguished and often only the conditions necessary to avoid operator aliasing are used to avoid aliasing artifacts (Gray, 1992; Bevc and Claerbout, 1992; Lumley et al., 1994; Abma, 1998), or more rarely, only the conditions to avoid image aliasing (Hale, 1991). However, the distinction becomes important when exploiting a priori knowledge on the dip spectrum of the data, because in this case the image needs to be more densely sampled than the data to avoid image aliasing. In addition, the distinction is important for prestack data sets that have larger dimensionality, and consequently a different sampling, than the image.

\section{THE ANTI-ALIASING DILEMMA}

Image-space aliasing can be corrected during processing without losing resolution by decreasing the spatial sampling of the image, because the image sampling is a processing parameter. In contrast, operator aliasing can only be avoided by suppressing some high-frequency components from the image, because the data sampling depends 
on acquisition parameters. Unfortunately this lowpass filtering decreases the image resolution. We therefore face a dilemma when imaging aliased data: on one side we need to apply anti-aliasing filters to avoid aliasing noise, on the other side we do not want to lose image resolution. Figure 1 exemplifies the problem. It shows the results of 3-D zero-offset time migration of a salt-dome flank in the Gulf of Mexico. The section on the left (Figure 1a) was 3-D migrated without applying any anti-aliasing filter, while the section on the right (Figure 1b) was obtained by applying a standard anti-aliased migration. Whereas the image obtained without anti-aliasing is much noisier than the anti-aliased one, it also has higher resolution. In the shallow part of the section shown in Figure 1a, the aliasing noise is so strong that is impossible to appreciate the higher resolution of Figure 1a compared with Figure 1b. But when comparing zooms into the deeper part of the sections (Figure 2), it is apparent that by applying anti-aliasing we lose resolution. In particular, the high-frequency dipping event at about $\mathrm{CMP} \mathrm{X}=700 \mathrm{~m}$ and Time $=2.2 \mathrm{~s}$ is poorly resolved in the anti-aliased migration (Figure 2b). The anti-aliased migration misses a whole wavelet cycle of the sediment truncation against the salt flank. If we consider that hydrocarbon reservoirs are often located at the sediment-salt interfaces, we appreciate the potential advantages of improving the resolution of events such as the sediment truncation shown in Figure 2.

Migration without anti-aliasing achieves higher resolution than the anti-aliased one because it images data components that are aliased in the data space. In particular, the steeply dipping energy reflected from the salt flanks visible in the data window shown in Figure 3 are aliased. Figure 4 shows the frequency-wavenumber spectrum of the data window in Figure 3. In addition to the central unaliased band of the spectrum, Figure 4 shows also the two spatially aliased bands on either side. The vertical black lines correspond to the Nyquist wavenumbers. The aliased dipping events correspond to a "cloud" in the spectrum that starts in the main band but crosses the positive Nyquist line and trespasses upon the aliased band. However, because there are no events dipping with negative time dips, the aliased components are still recoverable by the simple anti-aliasing method presented in this paper.

\section{ALIASING IN IMAGE SPACE}

The simplest type of aliasing related to imaging operator is image-space aliasing. It occurs when the spatial sampling of the image is too coarse to represent adequately the steeply dipping reflectors that the imaging operator attempts to build during the imaging process. In other words, image-space aliasing is caused by too coarse sampling of the image space, and consequent aliasing of the migration ellipsoid. A simple way of avoiding image aliasing is to make the spatial sampling of the image denser. But for a given spatial sampling of the image, to avoid image aliasing we need to limit, during the migration process, the frequency content of the image as a function of reflectors' dips. This goal can be accomplished by performing a dip-dependent temporal lowpass filtering of the input data during the summation process. An efficient 
Figure 1: 3-D migrations of a saltdome flank in the Gulf of Mexico: (a) migration obtained without any anti-aliasing filter, (b) migration obtained with the application of a "standard" anti-aliasing filter. biondo2-Comp-WL-intro [CR]

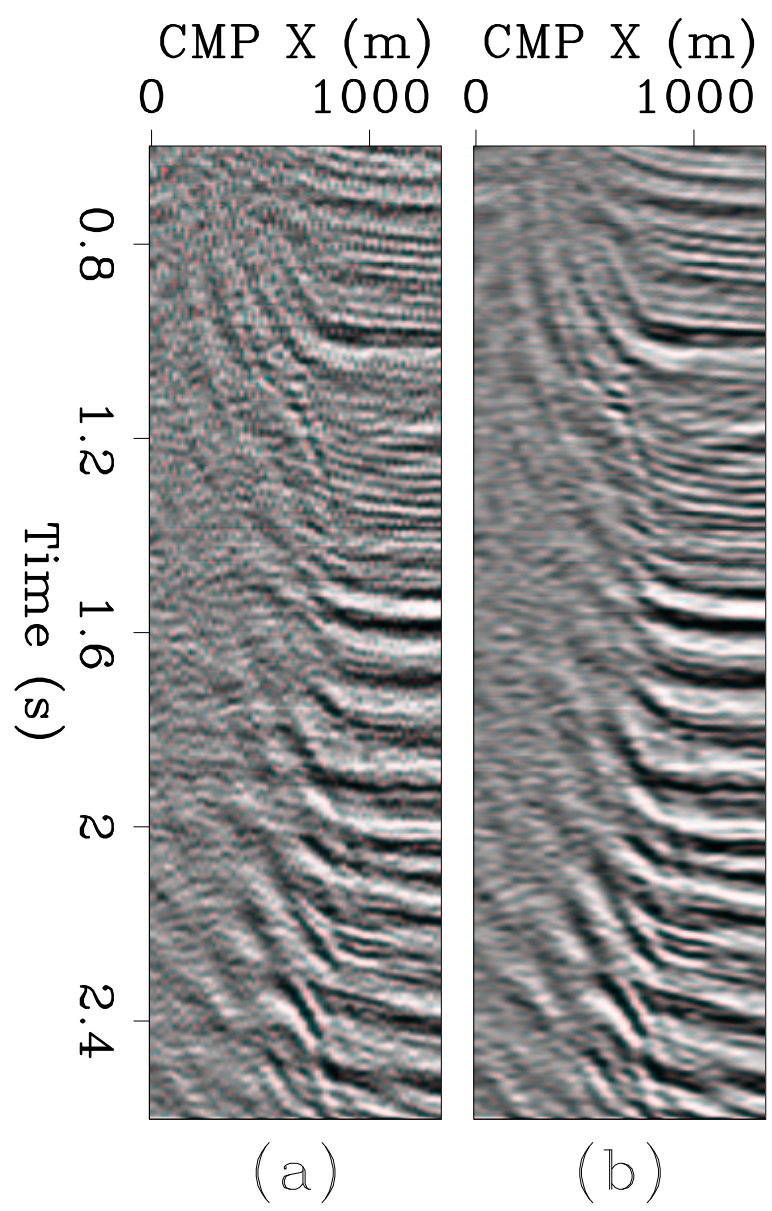

Figure 2: Zoom into the 3-D migrations of a salt-dome flank in the Gulf of Mexico shown in Figure 1: (a) migration obtained without any anti-aliasing filter, (b) migration obtained with the application of a "standard" anti-aliasing filter. biondo2-Comp-WB-intro [CR]

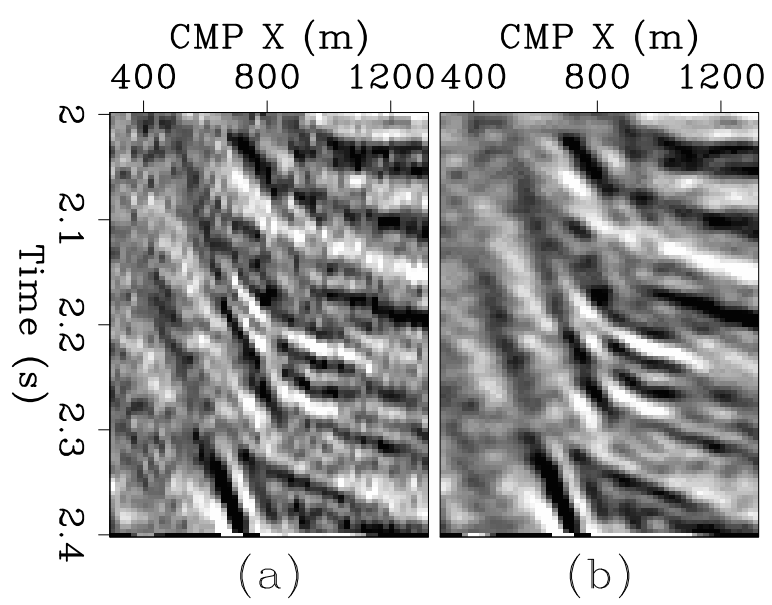


Figure 3: Data window containing aliased reflections from the salt flanks. biondo2-Wind-data [ER]



Figure 4: Frequency-wavenumber spectrum of the data window shown in Figure 3. Notice that the aliased events correspond to a "cloud" in the spectrum that starts in the main band but crosses the positive Nyquist line and trespasses upon the aliased band. biondo2-Wind-spec [ER] 
method to lowpass time-domain data with variable frequency is the triangle-filters method described in Basic Earth Imaging (Claerbout, 1995). An alternative method is to precompute lowpassed versions of the input traces, and select the appropriate input data during summation (Gray, 1992). This second method is potentially more accurate than the triangle filtering, and it is more computationally efficient when each input trace is summed into many output traces, as happens for 3-D migrations (Abma, 1998). However, it may require storing many versions of the input data. To reduce the storage requirements, without affecting the accuracy, I linearly interpolate the lowpassed traces along the frequency axis during the summation.

The anti-aliasing constraints to avoid image aliasing can be easily derived from basic sampling theory. For the case of time migration, when the coordinates of the image space are $\vec{\xi}=\left(\tau_{\xi}, x_{\xi}, y_{\xi}\right)$, the pseudo-depth frequency $\omega_{\tau}^{\xi}$ must fulfill the following inequalities:

$$
\begin{aligned}
\omega_{\tau}^{\xi} & \leq \frac{\pi}{\Delta x_{\xi} p_{x}^{\xi}} \\
\omega_{\tau}^{\xi} & \leq \frac{\pi}{\Delta y_{\xi} p_{y}^{\xi}}
\end{aligned}
$$

where $\Delta x_{\xi}$ and $\Delta y_{\xi}$ are respectively the image sampling rate of the $x_{\xi}$ and $y_{\xi}$ axes, and $p_{x}^{\xi}$ and $p_{y}^{\xi}$, are the reflector dip components.

As discussed above, it is convenient to apply an anti-aliasing filter as a band-pass filter of the input traces, before summing their contributions to the image into the output. Therefore, we need to recast the constraints on the pseudo-depth frequency $\omega_{\tau}^{\xi}$ of equations (1) into constraints on the input data frequency $\omega_{t}^{D}$. This distinction is important because the frequency content of the seismic wavelet changes during the imaging process because of stretching, or compression, of the time axis. In particular, during migration the wavelet is always stretched; neglecting this stretch would lead to anti-aliasing constraints that are too stringent. Notice, that the seismic wavelet may get compressed, instead of stretched, by other imaging operators, such as inverse DMO and AMO (Biondi et al., 1998). The pseudo-depth frequency of the image and the temporal frequency of the data are thus linked by the wavelet-stretch factor $d t_{D} / d \tau_{\xi}$, as $\omega_{\tau}^{\xi}=\omega_{t}^{D} d t_{D} / d \tau_{\xi}$. Taking into account the wavelet-stretch factor, we can write the constraints on the data frequency that control image aliasing, as a function of the image sampling rates $\Delta x_{\xi}$ and $\Delta y_{\xi}$, the image dips $p_{x}^{\xi}$ and $p_{y}^{\xi}$, and the wavelet-stretch factor $d t_{D} / d \tau_{\xi}$,

$$
\begin{aligned}
\omega_{t}^{D} & \leq \frac{\pi}{\Delta x_{\xi} p_{x}^{\xi} \frac{d t_{D}}{d \tau_{\xi}}} \\
\omega_{t}^{D} & \leq \frac{\pi}{\Delta y_{\xi} p_{y}^{\xi} \frac{d t_{D}}{d \tau_{\xi}}}
\end{aligned}
$$




\section{Image anti-aliasing for $3-\mathrm{D}$ prestack time migration}

For 3-D prestack time migration, the reflectors dips $p_{x}^{\xi}$ and $p_{y}^{\xi}$, and the wavelet-stretch factor $d t_{D} / d \tau_{\xi}$, can be analytically derived as functions of the input and output trace geometry and the input time. Appendix A presents the derivation of the analytical relationships that I apply for the following examples.

To gain intuition about the effects of incorporating an anti-aliasing filter in migration operator, it is instructive to analyze the images generated by migrating one single input trace into a cube. Figure 5 shows the result of migrating one trace without the application of any anti-aliasing filter. The input trace was recorded at an offset of $2.4 \mathrm{~km}$. The image sampling was $20 \mathrm{~m}$ in each direction $\left(\Delta x_{\xi}=\Delta y_{\xi}=20 \mathrm{~m}\right)$. Strong aliasing artifacts are visible in both the time slices and the vertical section.

Figure 6 shows the result of migrating the same data trace with an appropriate anti-aliased operator. Notice that the aliasing artifacts disappear as the frequency content of the imaged reflectors progressively decreases as the dips increase. Figure 7 shows the result of migrating the same data trace when the effects of the wavelet stretch are not taken into account; that is, by setting $d t_{D} / d \tau_{\xi}=1$. In this case the anti-aliasing filter over-compensates for the image dip and valuable resolution is lost at steep dips. Examining the time slice shown on the top of Figure 7, we notice that the loss of resolution is larger for regions of the migration ellipses with a steep dip along the cross-line direction.

Figure 8 shows the effects of image-space aliasing on the migrated results from the salt-dome data set shown in Figure 1. Figure 8a shows the non-antialiased migration results with $\Delta x_{\xi}=36 \mathrm{~m}$; that is the original sampling of the zero-offset data. Shallow dipping reflectors and the steep high-frequency event at about 2.2 seconds are badly aliased. The quality of the image improves by halving the image spatial sampling to $\Delta x_{\xi}=18 \mathrm{~m}$ (Figure $8 \mathrm{~b}$ ). It is useful to notice that the traces in Figure $8 \mathrm{a}$ are exactly the same as the odd traces in Figure 8b. Therefore, image aliasing does not add noise to the image, it just makes the image more difficult and ambiguous to interpret. The application of the image-space anti-aliasing constraints, expressed in equation (2), further improves the image quality, in particular for the shallower events (Figure 8c). Although the image in Figure 8c is less noisy than the images in Figure $8 \mathrm{a}$ and Figure 8b, it is still contaminated by aliasing artifacts. These artifacts are caused by operator aliasing, or data-space aliasing. Next section analyzes the causes of operator aliasing, and presents anti-aliasing constraints to eliminate it.

\section{OPERATOR ALIASING}

The previous section analyzed aliasing in the image space, that occurs when the spreading representation of the imaging operator is aliased. Data-space aliasing, the dual of image-space aliasing, occurs when the gathering representation of the imaging operator is aliased in the data space. This data-space aliasing is commonly 
Figure 5: Image obtained by applying Kirchhoff migration without anti-aliasing. biondo2-Imp-noantialias [ER]
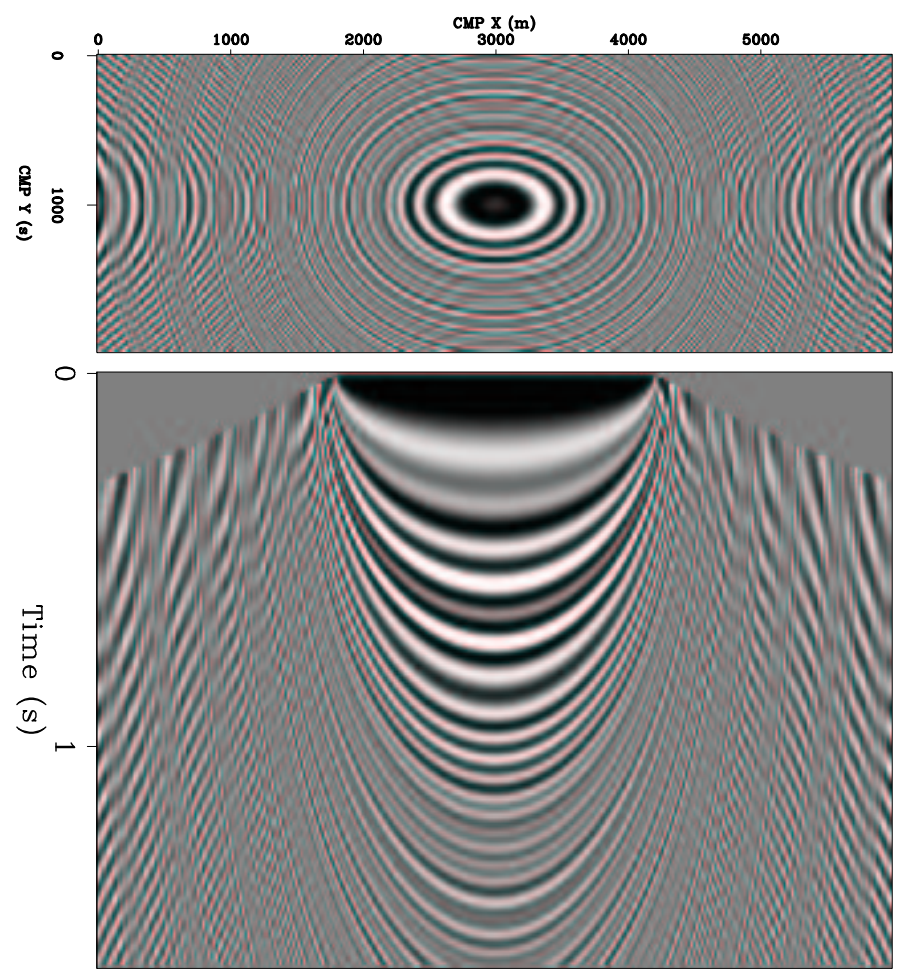

Figure 6: Image obtained by applying Kirchhoff migration with image-space anti-aliasing. biondo2-Imp-antialias-equal [ER]
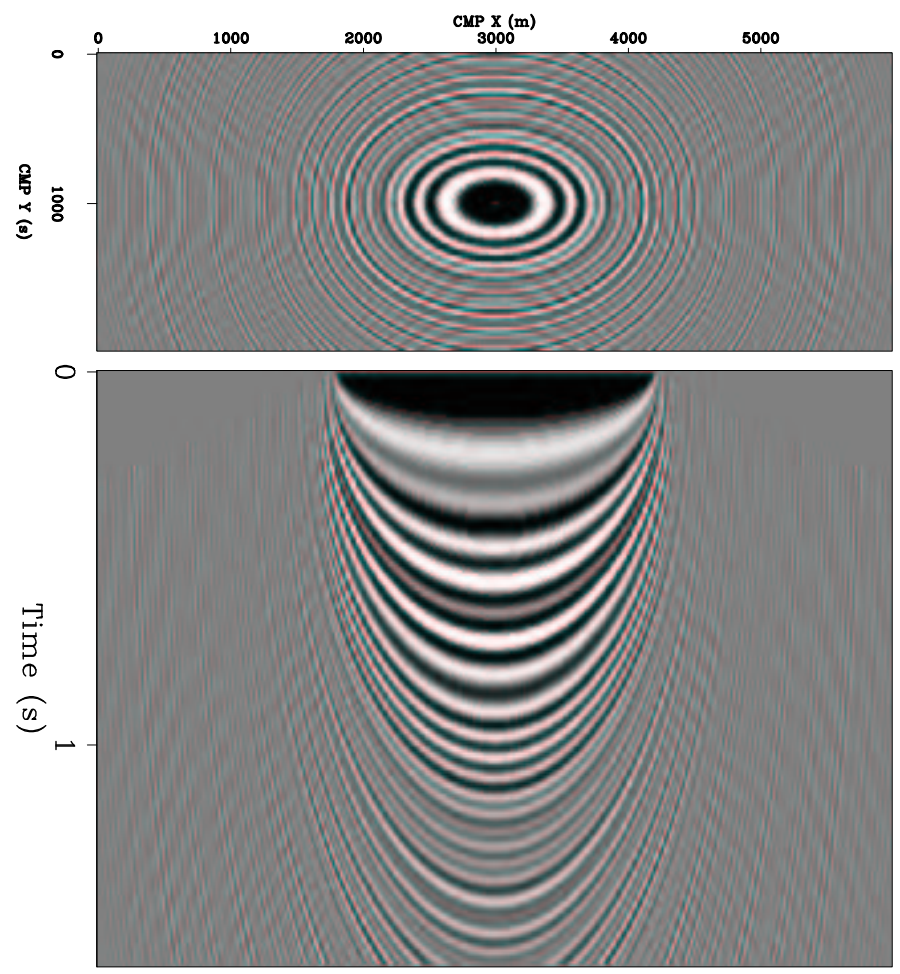


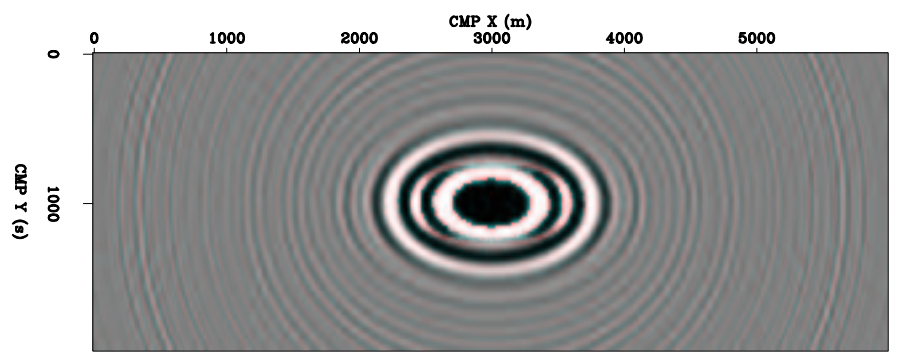

Figure 7: Image obtained by applying too strong of antialiasing by ignoring the effects of the wavelet stretch on the frequency content of the image. biondo2-Imp-nostretch [ER]

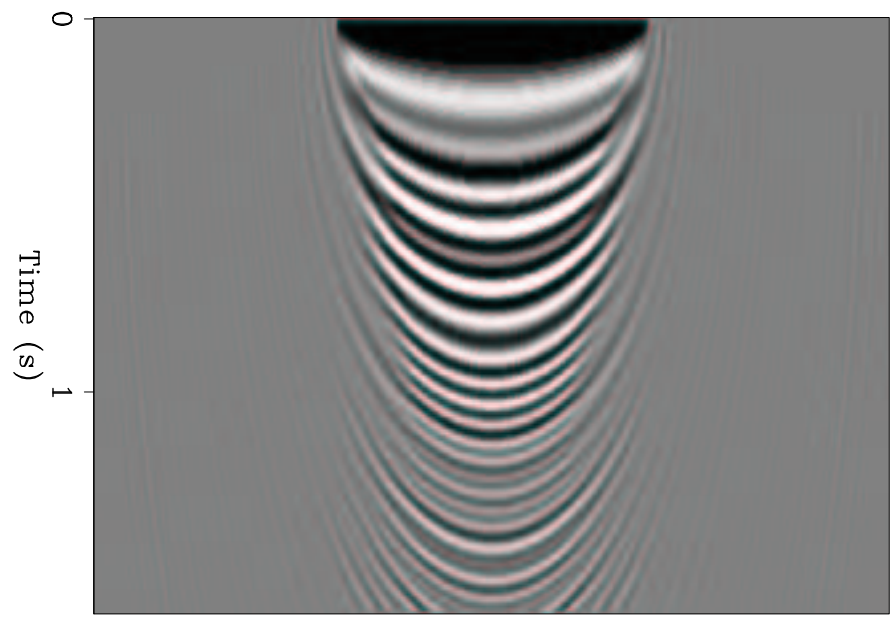

called operator aliasing. When operator aliasing occurs, noise is actually added to the image, in contrast to the image-space aliasing case. The next set of figures will illustrate the issues related to operator aliasing by analyzing a simple example in which the data are plane waves and the summation operator is a slant stack.

Figure 9 and Figure 10 show two plane waves. The plane waves are adequately sampled when the waveform is a $30 \mathrm{~Hz}$ sinusoid (Figure 9a), but the one with positive time dip is aliased when the waveform is a $60 \mathrm{~Hz}$ sinusoid (Figure 10a). The data aliasing can be observed both in the time-space domain, where the data appears to be dipping in the opposite direction, and in the wavenumber domain. The corresponding spatial spectra are shown at the bottom of the Figures. The solid lines correspond to the positive-dip plane wave, and the dotted line to the negative-dip plane wave. The spectrum for the positive-dip plane wave (solid line) at the bottom of Figure 9a shows two spikes at $\pm .75 k_{N}$ replicated at $\pm 1.25 k_{N}$ and $\pm 2.75 k_{N}$. Because of the doubling of the temporal frequency, in the spectrum at the bottom of Figure 10a the aliased spikes at $\pm 1.25 k_{N}$ moved into the central band to $\pm .5 k_{N}$.

Data summation along a given trajectory is equivalent to a two-step process; first the data are shifted to align the events along the desired trajectory. Second, the traces are stacked together. In the case of the slant-stack operator, the summation trajectories are lines and the first step is equivalent to the application of linear moveout (LMO) with the desired dip. Figure $9 \mathrm{~b}$ and Figure 10b show the results of applying LMO with the slowness of the positive-dip plane wave to the corresponding data in Figure $9 \mathrm{a}$ and Figure 10a. The traces on the right side of the sections are the results 


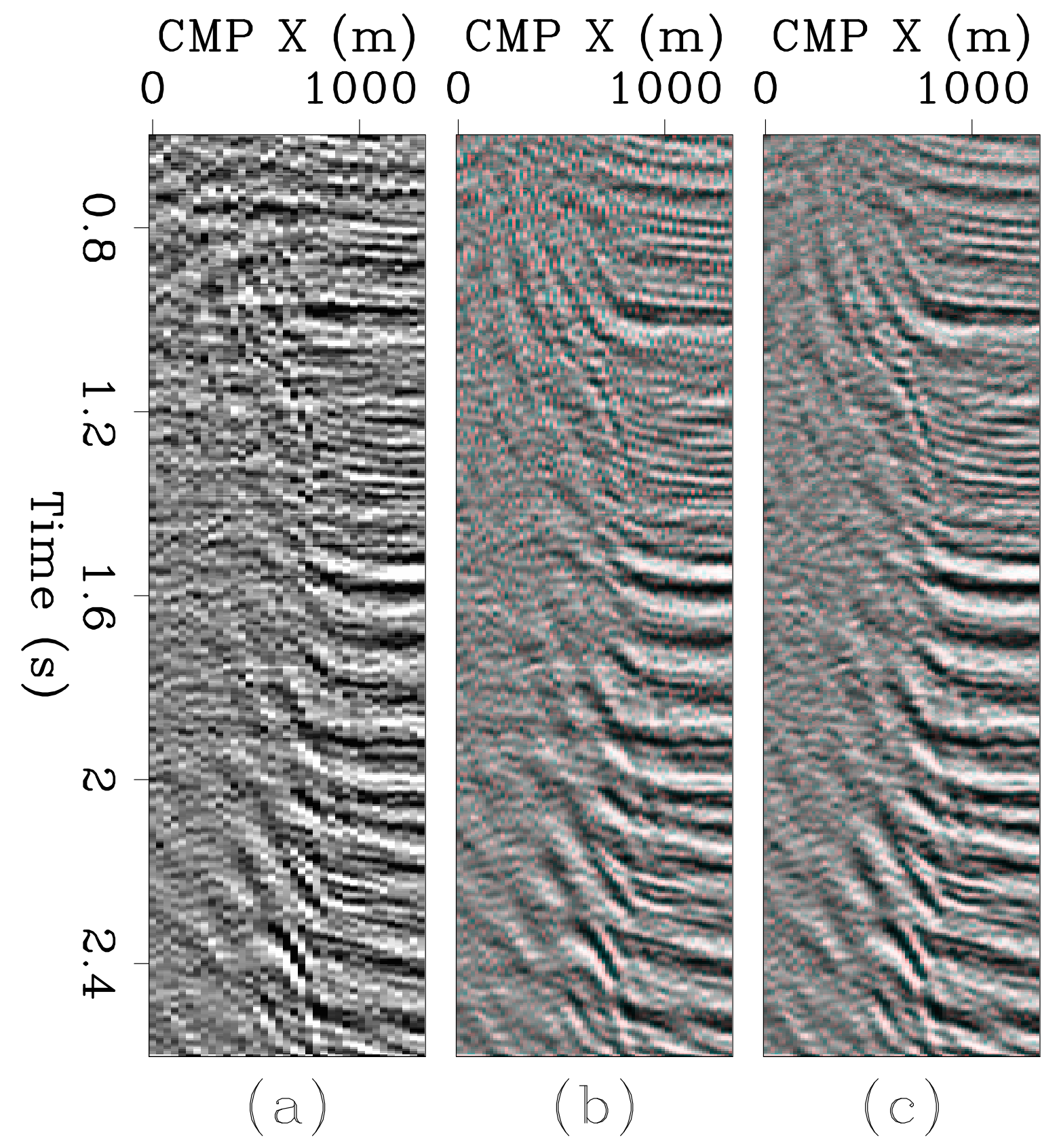

Figure 8: Migrated section from the Gulf of Mexico salt dome: (a) $\Delta x_{\xi}=36 \mathrm{~m}$ and no anti-aliasing, (b) $\Delta x_{\xi}=18 \mathrm{~m}$ and no anti-aliasing, (c) $\Delta x_{\xi}=18 \mathrm{~m}$ and image-space anti-aliasing. biondo2-Comp-noanti-mod [CR] 
of stacking the corresponding data. At $30 \mathrm{~Hz}$ no aliasing occurs, and after LMO only the original plane wave stacks coherently, as desired. In contrast, at $60 \mathrm{~Hz}$ both plane waves stack coherently after LMO as well as the original plane wave. In general, artifacts are generated when data that are not aligned with the summation path stack coherently into the image. This phenomenon is the cause of the aliasing noise that degrades the image when operator aliasing occurs. To avoid adding aliasing noise to the image we could lowpass filter the input data according to the operator dips. The resulting anti-aliasing constraints are:

$$
\begin{aligned}
\omega_{t}^{D} & \leq \frac{\pi}{\Delta x_{D} p_{x}^{\mathrm{op}}} \\
\omega_{t}^{D} & \leq \frac{\pi}{\Delta y_{D} p_{y}^{\mathrm{op}}}
\end{aligned}
$$

where $\Delta x_{D}$ and $\Delta y_{D}$ are the sampling rates of the data axes, and $p_{x}^{\text {op }}$ and $p_{y}^{\text {op }}$ are the operator dips. These, or equivalent, relationships have been presented by a number of authors (Gray, 1992; Bevc and Claerbout, 1992; Lumley et al., 1994; Abma, 1998). Although, these constraints may be correct for several important cases, they do not take into account the fact that operator aliasing depends on the presence of conflicting dips in the data, as shown by the previous example. More precisely, it depends on the dip bandwidth in the data.

To further examine the idea of operator aliasing depending on the dip bandwidth in the data, we consider the two plane waves shown in Figure 11. In this case the two plane waves have a $60 \mathrm{~Hz}$ waveform, as in Figure 10, but with the second plane wave flat instead of dipping with a negative time dip. The two plane waves have conflicting dips; but the additional plane wave does not interfere with the stacking of the original plane wave even with a $60 \mathrm{~Hz}$ waveform.

The last two examples demonstrate that the limits on the dip range for unaliased summation paths are a direct function of the expected dips in the data along the summation axes. If $\left(p_{x}^{\min }, p_{y}^{\min }\right)$ and $\left(p_{x}^{\max }, p_{y}^{\max }\right)$ are respectively the minimum and maximum dips expected in the data, then, to avoid operator aliasing, the operator dip must fulfill the following inequalities:

$$
\begin{gathered}
p_{x}^{\max }-\frac{2 \pi}{\omega_{t}^{D} \Delta x} \leq p_{x}^{\mathrm{op}} \leq p_{x}^{\mathrm{min}}+\frac{2 \pi}{\omega_{t}^{D} \Delta x} \\
p_{y}^{\max }-\frac{2 \pi}{\omega_{t}^{D} \Delta y} \leq p_{y}^{\mathrm{op}} \leq p_{y}^{\mathrm{min}}+\frac{2 \pi}{\omega_{t}^{D} \Delta y}
\end{gathered}
$$

The inequalities expressed in equation (4) can be easily recast as anti-aliasing constraints on the maximum frequency in the data as:

$$
\begin{aligned}
\omega_{t}^{D} & \leq \frac{2 \pi}{\Delta x\left(p_{x}^{\mathrm{op}}-p_{x}^{\mathrm{min}}\right)} \\
\omega_{t}^{D} & \leq \frac{2 \pi}{\Delta x\left(p_{x}^{\mathrm{max}}-p_{x}^{\mathrm{op}}\right)},
\end{aligned}
$$



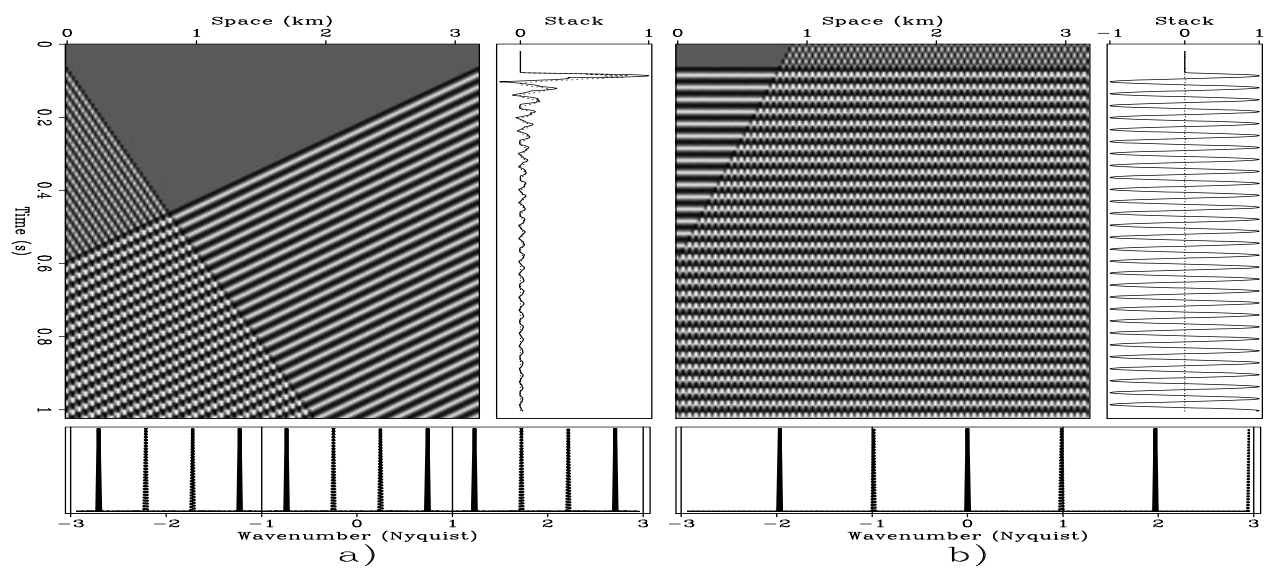

Figure 9: Two plane waves with dips of $.5 \mathrm{~s} / \mathrm{km}$ and $.166 \mathrm{~s} / \mathrm{km}$ and $30 \mathrm{~Hz}$ waveform, a) before linear moveout and b) after linear moveout. biondo2-Fig-30-sec [ER]
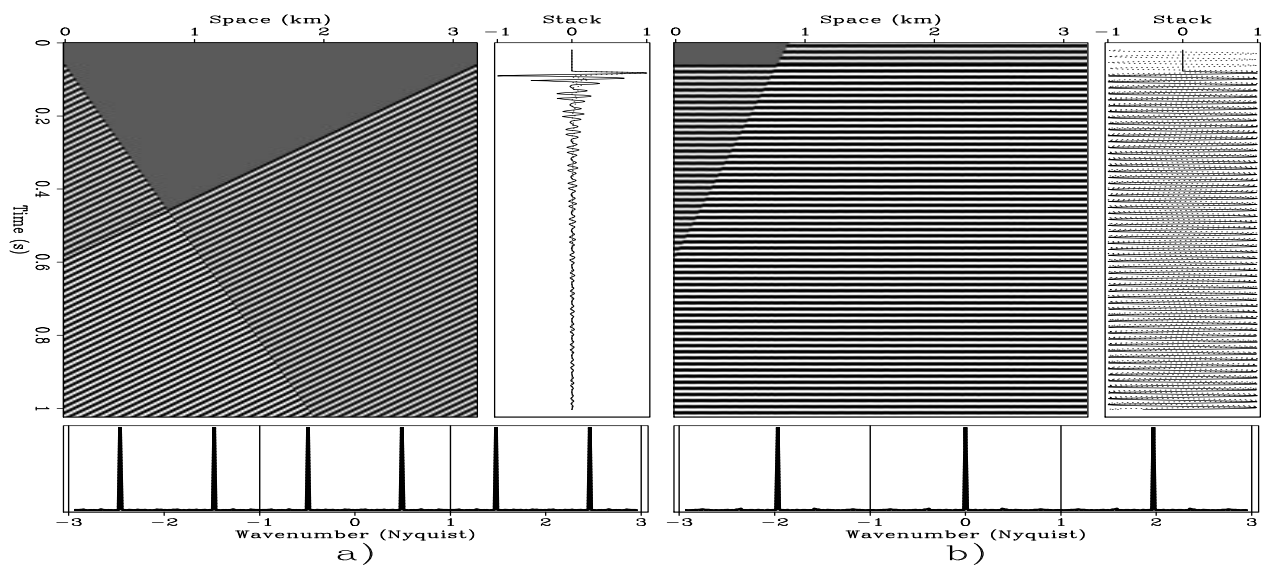

Figure 10: Two plane waves with dips of $.5 \mathrm{~s} / \mathrm{km}$ and $.166 \mathrm{~s} / \mathrm{km}$ and $60 \mathrm{~Hz}$ waveform, a) before linear moveout and b) after linear moveout. biondo2-Fig-60-sec [ER]
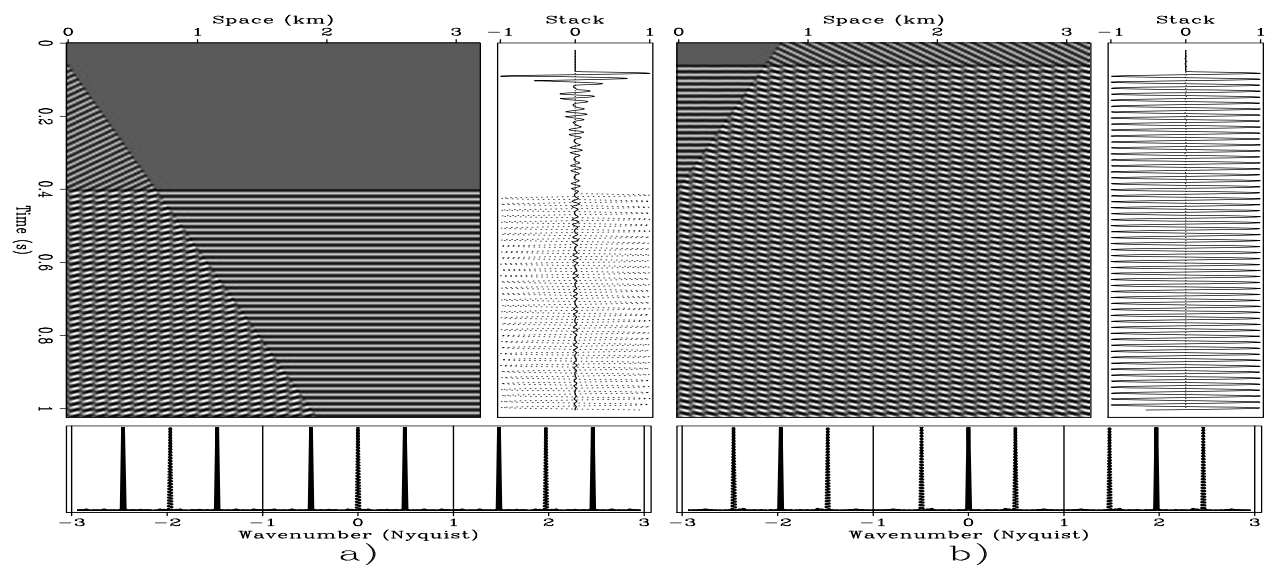

Figure 11: Two plane waves with dips of $.5 \mathrm{~s} / \mathrm{km}$ and $0 \mathrm{~s} / \mathrm{km}$ and $60 \mathrm{~Hz}$ waveform, a) before linear moveout and b) after linear moveout. biondo2-Fig-60-gath [ER] 


$$
\begin{aligned}
\omega_{t}^{D} & \leq \frac{2 \pi}{\Delta y\left(p_{y}^{\mathrm{op}}-p_{y}^{\min }\right)}, \\
\omega_{t}^{D} & \leq \frac{2 \pi}{\Delta y\left(p_{y}^{\max }-p_{y}^{\mathrm{op}}\right)} .
\end{aligned}
$$

The constraints expressed in equation (5) can be used as alternatives, or in conjunction with the constraints expressed in equation (3) to antialias summation operators. Examining the inequalities expressed in equation (4), we can notice that the two sets of constraints are equivalent when, for each frequency, the data dip limits $p^{\text {min }}$ and $p^{\max }$ are set to $k_{N y q} / \omega_{t}^{D}$, where $k_{N y q}$ is the Nyquist wavenumber along each axis. That is, if we assume that there is no spatial aliasing in the data, the constraints expressed in equation (5) are equivalent to the constraints expressed in equation (3).

The data-dips limits $\mathbf{p}^{\min }$ and $\mathbf{p}^{\max }$ can be both spatially and time varying according to the expected local dips in the data. Therefore, the anti-aliasing filtering applied to the data as a consequence of the constraints in equation (5) can be fairly complex, and dependent on: local dips, time, and spatial coordinates. If no a priory knowledge on the local dips is available, and the summation is carried out along the midpoint axes, twice the inverse of propagation velocity is a reasonable bound on the absolute values of both $\mathbf{p}^{\text {min }}$ and $\mathbf{p}^{\max }$. In contrast, in the case that the summation is performed along the offset axes, as for CMP stacks, $\mathbf{p}^{\text {min }}$ can be safely assumed to be positive, and at worst equal to zero. In practice the bounds on the data's expected dips should take into account all types of events, and not only the dips of the reflections that we aim to image. For example, in CMP gathers recorded on land, $p^{\max }$ should take into account low-velocity events such as ground roll.

The most substantial benefits of applying the more general constraints expressed in equation (5) are achieved when asymmetric bounds on the dips in the data enable imaging without aliasing high-frequency components that are present in the data as aliased energy, and consequently would be filtered out if the constraints in equation (3) were applied. An important case when asymmetric bounds on the data dips are realistic is the imaging of steep salt-dome flanks, as in the Gulf of Mexico data set shown above. In this case, we can assume that the negative time dips in the data are small. According to the equations in (4), the increase in $p^{\text {min }}$ raises the limit on the maximum positive operator dip. In practice, the application of the generalized constraints in equation (5), when $\mathbf{p}^{\min } \neq \mathbf{p}^{\max }$ cause the migration operator to be asymmetric, with dip bandwidth dependent on reflector direction. Figure 12 and Figure 13 show an example of the effects of asymmetric dip bounds on the migration operator. For both images, the image sampling is the same as in Figure $6\left(\Delta x_{\xi}=\Delta y_{\xi}=20 \mathrm{~m}\right)$, but the data sampling is assumed to be coarser than the image sampling by a factor of two; that is, $\Delta x_{\xi}=\Delta y_{\xi}=40 \mathrm{~m}$. When the constraints in equation (3) are applied (see Figure 12), the operator has lower resolution than in Figure 6. But if we assume that $p_{x}^{\min }=0$, and apply the constraints in equation (5) (see Figure 13), the positive time dips are imaged with the same resolution as in Figure 6. 
Figure 12: Image obtained by

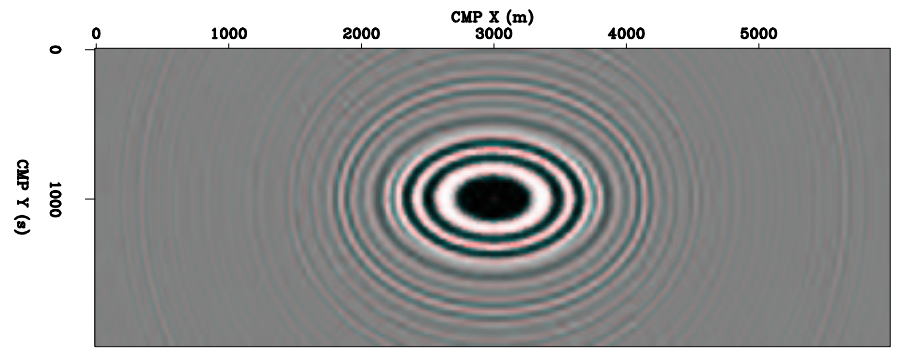
applying Kirchhoff migration with "standard" anti-aliasing. Sampling rates are: $\Delta x_{\xi}=\Delta y_{\xi}=20 \mathrm{~m}$ and $\Delta x_{D}=\Delta y_{D}=40 \mathrm{~m}$. biondo2-Imp-antialias-nodirect [ER]

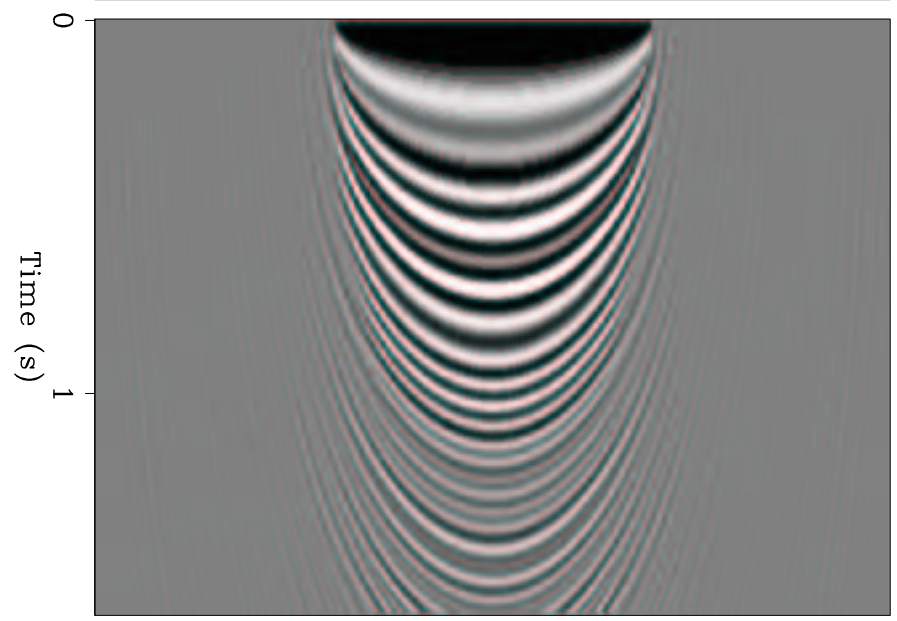

Figure 13: Image obtained by applying Kirchhoff migration with "directed" anti-aliasing assuming $p_{x}^{\min }=0$. Sampling rates are: $\Delta x_{\xi}=\Delta y_{\xi}=20 \mathrm{~m}$ and $\Delta x_{D}=\Delta y_{D}=40 \mathrm{~m}$. biondo2-Imp-antialias-direct [ER]
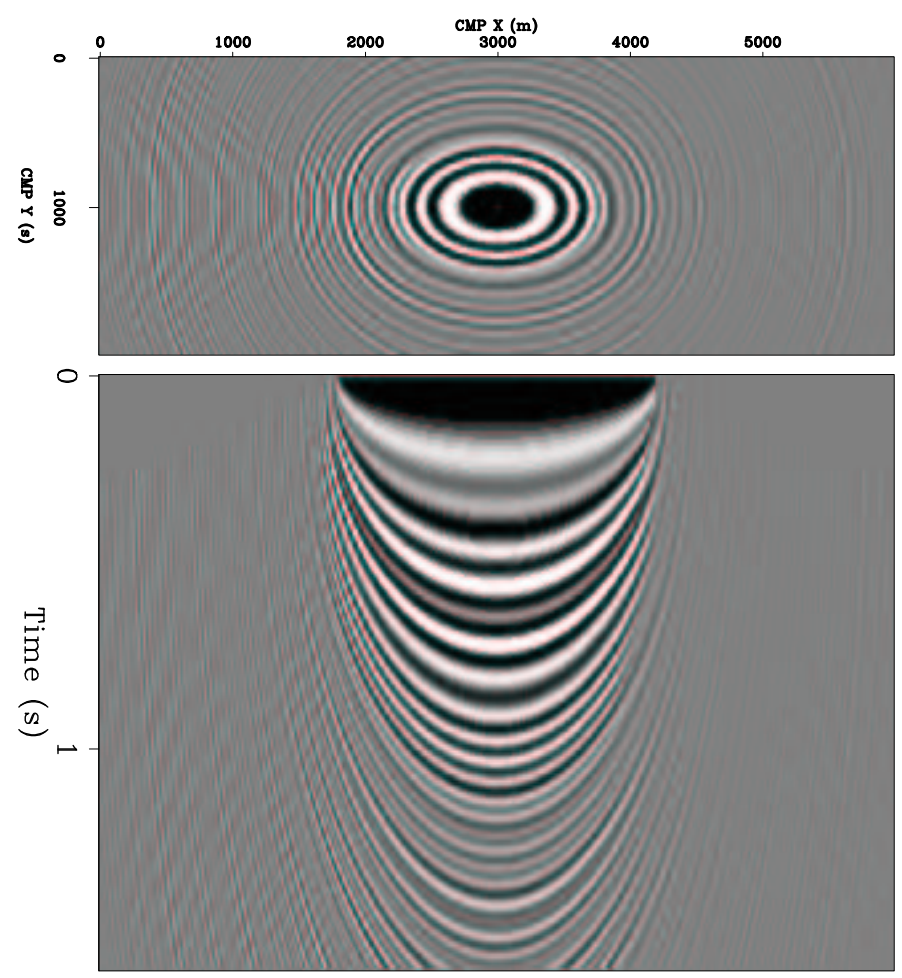


\section{Link between operator anti-aliasing and image anti-aliasing}

The constraints on the data frequency to avoid operator aliasing and image aliasing are different in general; and both sets of constraints must be respected to assure a high-quality image. However, the two sets of constraints are equivalent in many practical situations. The analysis is simple for zero-offset or constant-offset data, where we can assume that the summation surfaces $t_{D}=t_{D}\left(\vec{\xi}, x_{m}, y_{m}\right)$ are only functions of the image point coordinates $\vec{\xi}$ and of the midpoint coordinates of the data trace $\left(x_{m}, y_{m}\right)$.

The first condition for linking image aliasing to operator aliasing is that the data are not spatially aliased, and thus the operator anti-aliasing constraints are the ones expressed in equation (3), and not the constraints expressed in equation (5). Comparing the constraints for operator anti-aliasing [equation (3)] with the constraints for image anti-aliasing [equation (2)] we can easily notice that a necessary condition for them being uniformly equivalent is that the data sampling rates $\Delta x_{D}$ and $\Delta y_{D}$ must be equal to the image sampling rates $\Delta x_{\xi}$ and $\Delta y_{\xi}$. The other necessary conditions are that $p_{x}^{\mathrm{op}}=p_{x}^{\xi} d t_{D} / d \tau_{\xi}$ and $p_{y}^{\mathrm{op}}=p_{y}^{\xi} d t_{D} / d \tau_{\xi}$. These conditions are fulfilled in the important case of spatially invariant imaging operators, as it can be shown by applying the chain rule to the derivative of the summation surfaces $t_{D}$ with respect to the midpoint coordinates of the data trace $\left(x_{m}, y_{m}\right)$ :

$$
\begin{aligned}
& p_{x}^{\mathrm{op}}=\frac{d t_{D}}{d x_{m}}=\frac{d t_{D}}{d z_{\xi}} \frac{d z_{\xi}}{d x_{\xi}} \frac{d x_{\xi}}{d x_{m}}=\frac{d t_{D}}{d z_{\xi}} p_{x}^{\xi} \\
& p_{y}^{\mathrm{op}}=\frac{d t_{D}}{d y_{m}}=\frac{d t_{D}}{d z_{\xi}} \frac{d z_{\xi}}{d y_{\xi}} \frac{d y_{\xi}}{d y_{m}}=\frac{d t_{D}}{d z_{\xi}} p_{y}^{\xi} .
\end{aligned}
$$

The equalities in (6) rely on the horizontal invariance of the imaging operator, by requiring that the derivatives of the horizontal coordinates of the image point with respect to the horizontal coordinates of the data trace to be equal to one. For migration, these conditions are strictly fulfilled only in horizontally layered media, though they are approximately fulfilled when migration velocity varies smoothly. Because equalities in (6) do not require any other assumptions on the shape of the summation surfaces; the same link between operator aliasing and image aliasing exists for all spatially-invariant integral operators, such as DMO and AMO.

The distinction between operator aliasing and image aliasing can thus be safely ignored when time migrating well sampled zero-offset data (Bevc and Claerbout, 1992; Lumley et al., 1994; Claerbout, 1995), but it ought be respected when depth migrating irregularly sampled prestack data. This distinction is also important when a priori assumptions on the dips in the data permit setting less stringent operator anti-aliasing constraints, and thus the reflectors can be imaged with high-resolution and without operator-aliasing artifacts.

An open, and more subtle, question remains regarding the operator aliasing of prestack data, that in general do not constitute a minimal data set (Padhi and Holley, 
1997). However, also in this case the constraints to avoid image aliasing [equation (2)] must be respected to produce high-quality and interpretable images.

\section{HIGH-RESOLUTION IMAGING OF SALT FLANKS}

The high-resolution imaging of salt-dome flanks is an important application of the theory described in the previous two sections. In this case we can often assume that in the proximity of the salt flanks the data contains no, or little, energy dipping in the direction opposite to the reflections from the flanks. According to the theory developed in the previous section, this assumption enables the imaging of the salt flanks with higher resolution than otherwise possible. However, it is important to be aware that when increasing the image frequency content by applying the constraints in equation (5) in place of the constraints in equation (3), we run the risk of aliasing the image. As discussed in the previous section, the conditions that avoid operator aliasing do not guarantee avoidance of image aliasing. Therefore, the constraints to avoid image aliasing [equation (2)] must also be taken into account, and the image sampling must be reduced to achieve both goals of avoiding image aliasing and preserving high-frequency components. Because of image aliasing considerations, the images of the salt flanks from the Gulf of Mexico data that are shown in the following Figures are sampled twice as densely $\left(\Delta x_{\xi}=\Delta y_{\xi}=18 \mathrm{~m}\right)$, as the zero-offset data $\left(\Delta x_{D}=\Delta y_{D}=36 \mathrm{~m}\right)$.

The first step to apply the high-resolution imaging method presented in this paper is to determine the appropriate values for the bounds on the data dips. For the sake of simplicity, for this example I choose constant bounds; that is, $p_{x}^{\min }=-.082 \mathrm{~s} / \mathrm{km}$, $p_{x}^{\max }=.48 \mathrm{~s} / \mathrm{km}, p_{y}^{\min }=-.8 \mathrm{~s} / \mathrm{km}, p_{y}^{\max }=.8 \mathrm{~s} / \mathrm{km}$. In more geologically-complex cases it may be advantageous to allow the data-dip bounds to vary both in time and space. Figure 14 shows the same data spectrum as Figure 4; the dashed line superimposed onto the spectrum cover the areas determined by the inequalities of equation (4), according to the chosen bounds for $p_{x}^{\min }$ and $p_{y}^{\max }$. The areas that honor all anti-aliasing constraints, and thus that represent data components that are used by the imaging, are covered by crossing dashed lines. A large swath of the aliased energy with positive time dips is used by the high-resolution imaging, whereas it would be discarded if standard anti-aliasing methods were used.

The improvements in image resolution that are made possible by the proposed anti-aliasing method are demonstrated in Figure 15- Figure 17. Figure 15a shows the results of 3-D post-stack migration without using any anti-aliasing. Figure 15b shows the results when the new anti-aliasing constraints in equation (5) are applied. And Figure 15c shows the results when the standard anti-aliasing constraints in equation (3) are applied. The differences between the results are better appreciated by comparing windows zooming into smaller parts of the sections. Figure 16 shows the comparison for the shallower part of the section. The image obtained without anti-aliasing is uninterpretable because of the strong aliasing noise. The image obtained with the proposed method shows better resolution of several dipping reflectors 


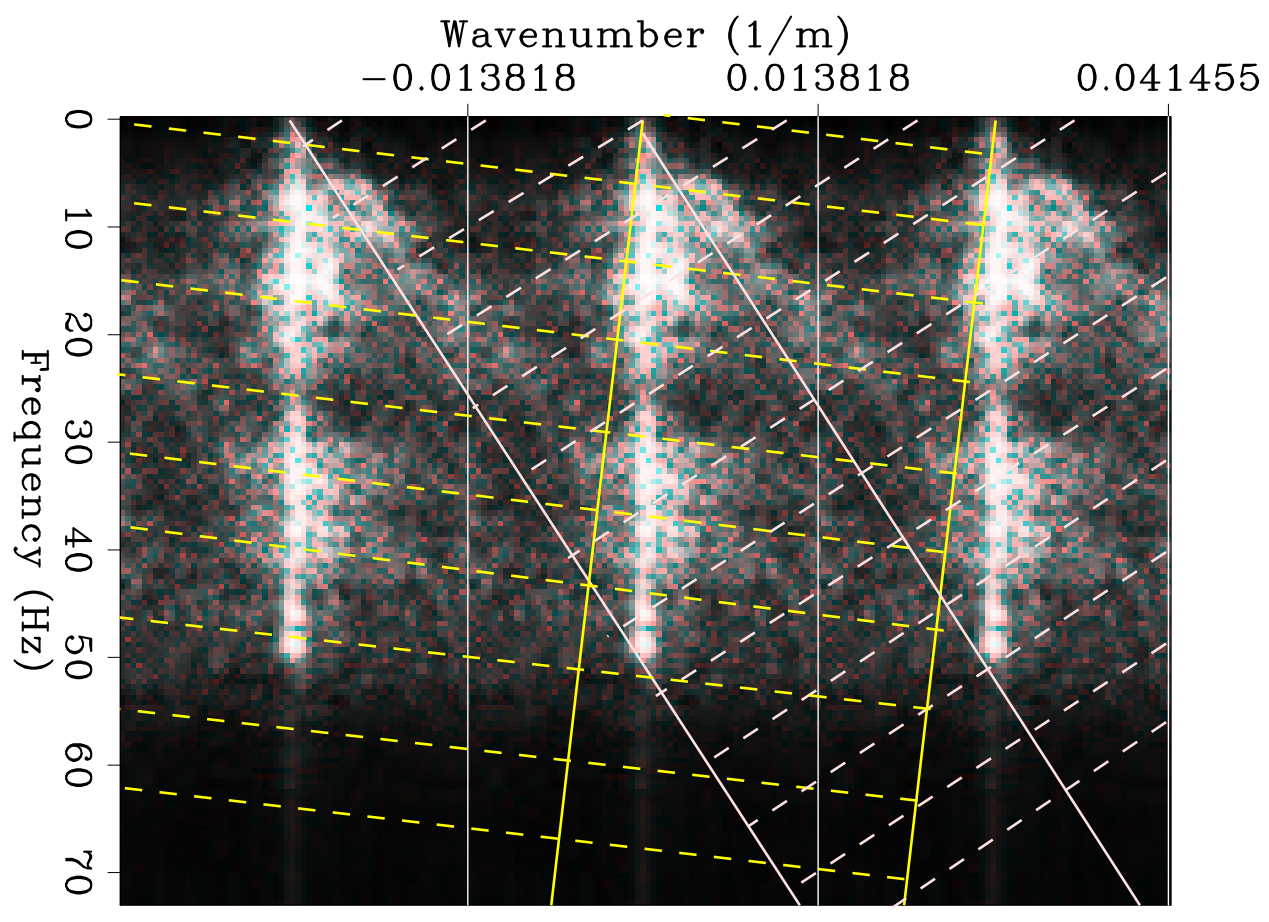

Figure 14: Frequency-wavenumber spectrum of a data window (same as in Figure 4). The dashed line superimposed onto the spectrum cover the areas determined by the inequalities of equation (4). The areas that respect all the operator anti-aliasing constraints, and thus that represent data components that are used by the imaging, are covered by dashed lines along both direction. biondo2-Wind-spec-ann [NR]

and of the steep salt flank. Figure 15 demonstrates (same area shown in Figure 1) that the high-frequency sediment truncation against the salt flank, (CMP X=700 m and Time $=2.2 \mathrm{~s}$ ) is well resolved in the image obtained using the proposed method, whereas it is poorly resolved in the image obtained using the traditional methods.

\section{CONCLUSIONS}

When imaging data affected by spatial aliasing, we would like not to be forced to trade-off resolution for signal-to-noise ratio. When a priori knowledge of the structure in the target zone enables us to make realistic assumptions on the dip content of the data, the application of the anti-aliasing method presented in this paper yields images with higher resolution than the images obtained by applying the standard anti-aliasing criteria. The new anti-aliasing method presented in this paper is based on a more comprehensive understanding of aliasing of Kirchhoff-like imaging operators.

Imaging of steeply dipping salt flanks is an important application of the proposed "high-resolution" method. The steeply dipping sediment terminations against the salt are important areas for hydrocarbon exploration, and in many cases it is realistic 


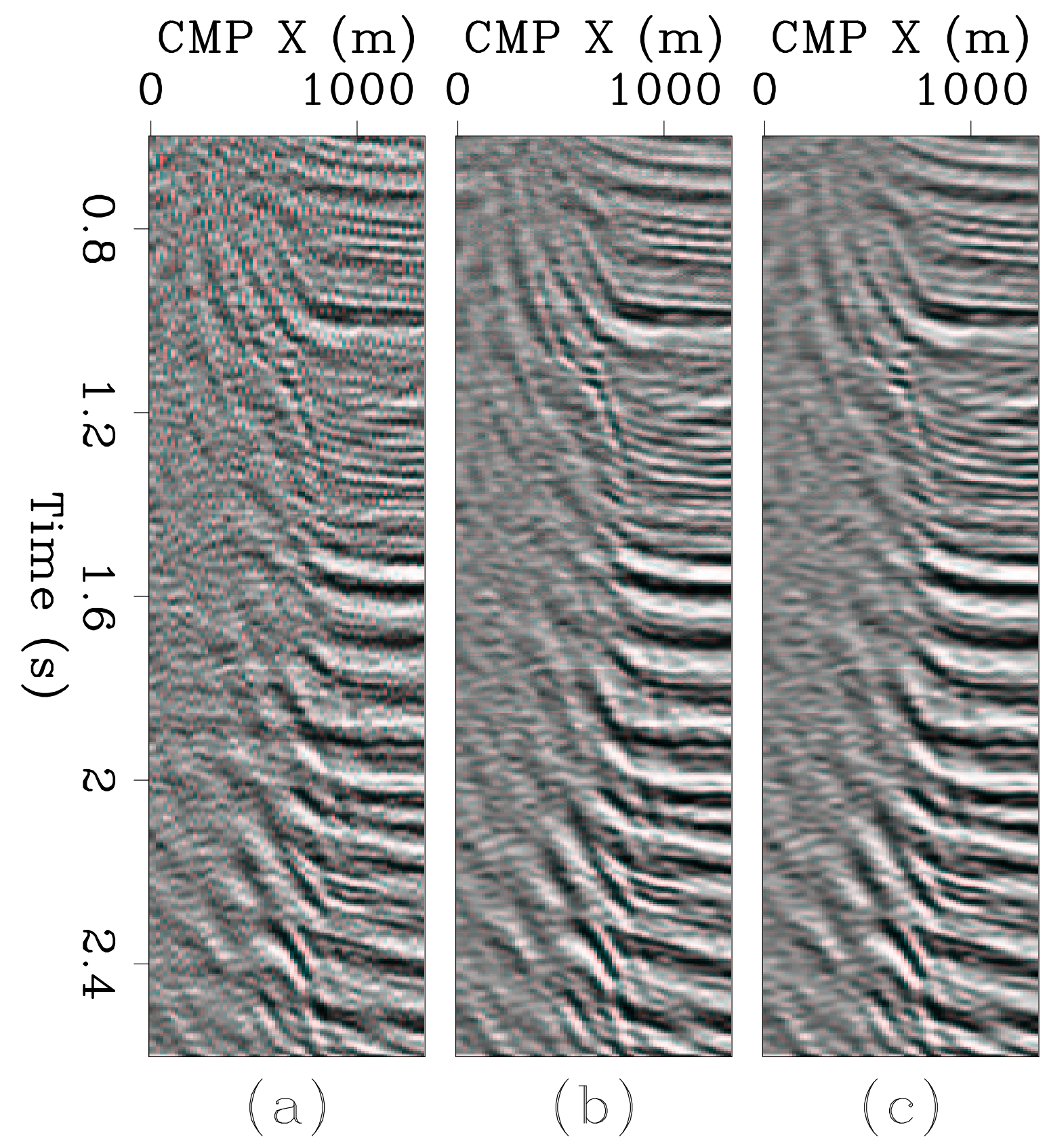

Figure 15: 3-D migrations of a salt-dome flank in the Gulf of Mexico: (a) migration obtained without any anti-aliasing filter, (b) migration obtained with the application of the proposed "high-resolution" anti-aliasing filter, (c) migration obtained with the application of a "standard" anti-aliasing filter. biondo2-Comp-WL-anti [CR] 


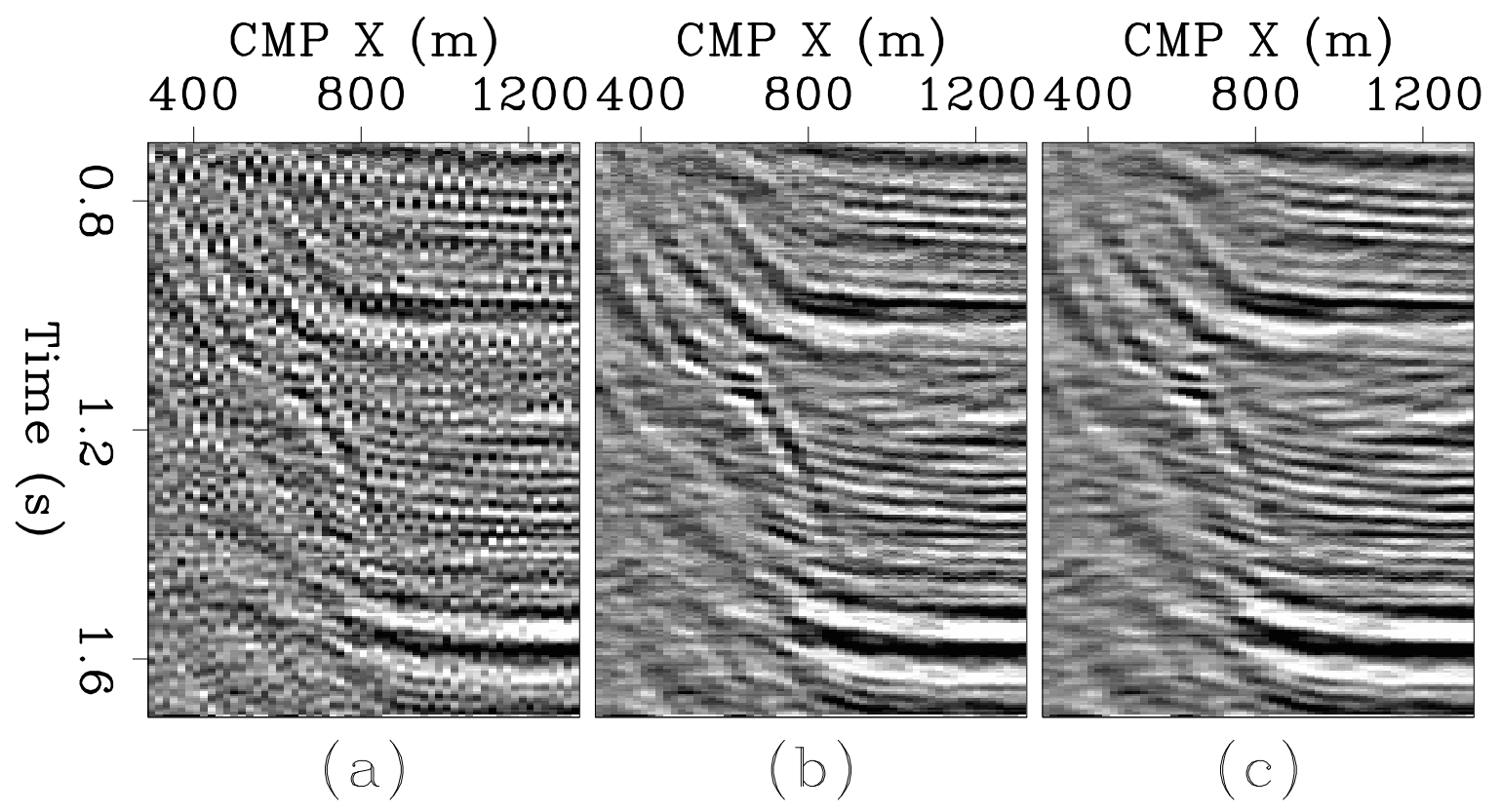

Figure 16: Zoom into the shallower part of the 3-D migrations of a salt-dome flank in the Gulf of Mexico shown in Figure 15: (a) migration obtained without any antialiasing filter, (b) migration obtained with the application of the proposed "highresolution" anti-aliasing filter, (c) migration obtained with the application of a "standard" anti-aliasing filter. biondo2-Comp-WT-anti [CR]

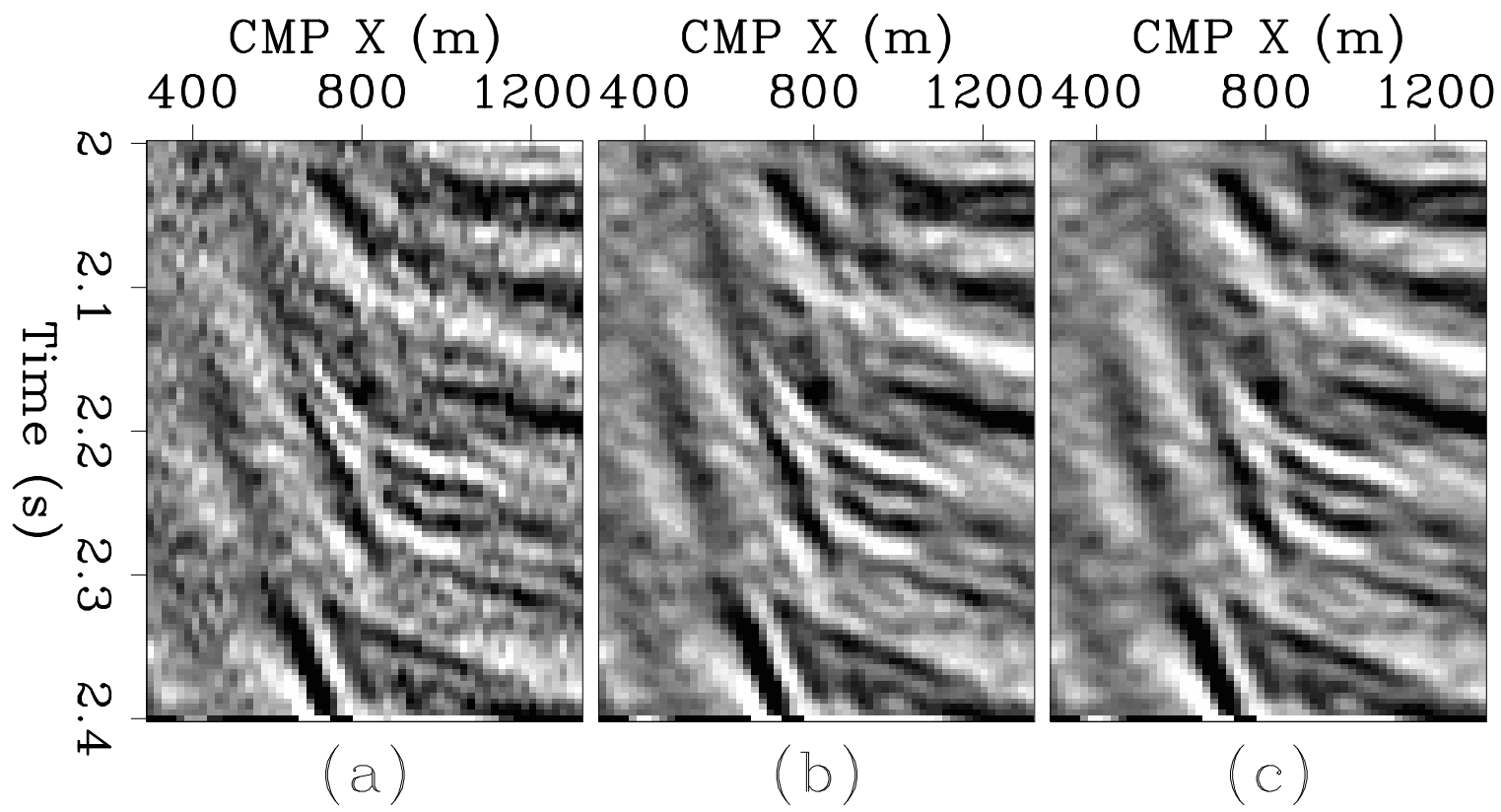

Figure 17: Zoom into the deeper part of the 3-D migrations of a salt-dome flank in the Gulf of Mexico shown in Figure 15: (a) migration obtained without any anti-aliasing filter, (b) migration obtained with the application of the proposed "high-resolution" anti-aliasing filter, (c) migration obtained with the application of a "standard" antialiasing filter. biondo2-Comp-WB-anti [CR] 
to assume that the data close to the salt flanks do not contain energy dipping in the direction opposite to the flank reflections. The data example from the Gulf of Mexico demonstrates that the proposed method improves image resolution, without adding aliasing noise.

\section{ACKNOWLEDGMENTS}

I would like to thank Unocal for releasing to SEP the data set shown in the paper. In particular, I would like to thank Wook Lee with Unocal for preparing and sending the data.

\section{REFERENCES}

Abma, R., 1998, Comparisons of anti-aliasing methods in Kirchhoff migration: submitted to Geophysics.

Bevc, D., and Claerbout, J., 1992, Fast anti-aliased Kirchhoff migration and modeling: SEP-75, 91-96.

Biondi, B., Fomel, S., and Chemingui, N., 1998, Azimuth moveout for 3-D prestack imaging: Geophysics, 63, no. 2, 574-588.

Brink, M., Gimse, I., Turner, P., Stolte, C., and Ronen, S., 1997, Resolution and coverage beyond traditional aliasing: 67th Ann. Internat. Meeting, Soc. Expl. Geophys., 1123-1126.

Chemingui, N., and Biondi, B., 1998, Discrete Kirchhoff theory and irregular geometry: SEP-97, 117-130.

Claerbout, J. F., 1995, Basic Earth Imaging: Stanford Exploration Project.

Crawley, S., 1998, Shot interpolation for radon multiple suppression: SEP-97, 173182.

Gray, S. H., 1992, Frequency-selective design of the Kirchhoff migration operator: Geophys. Prosp., 40, no. 5, 565-572.

Hale, D., 1991, A nonaliased integral method for dip moveout: Geophysics, 56, no. $6,795-805$.

Lumley, D. E., Claerbout, J. F., and Bevc, D., 1994, Anti-aliased Kirchhoff 3-D migration: 64th Ann. Internat. Meeting, Soc. Expl. Geophys., 1282-1285.

Neidell, N., 1997, Perceptions in seismic imaging: The Leading Edge, 16, no. 7,8,9,10.

Padhi, T., and Holley, T. K., 1997, Wide azimuth - why not?: The Leading Edge, 16, 175-177. 
Pica, A., 1996, Model-based anti-aliased Kirchhoff 3D PSDM: 58th Mtg. Eur. Assoc. Expl Geophys., Eur. Assoc. Expl. Geophys.

Wisecup, D., 1998, Unambiguous signal recovery above Nyquist using randomsample-interval imaging: Geophysics, 63, no. 2, 763-771.

\section{APPENDIX A}

\section{ANTI-ALIASING CONSTRAINTS FOR 3-D PRESTACK TIME MIGRATION}

For 3-D prestack time migration, the reflectors' dips $p_{x}^{\xi}$ and $p_{y}^{\xi}$, and the waveletstretch factor $d t_{D} / d \tau_{\xi}$, can be analytically derived as functions of the input and output trace geometry. Starting from the prestack time-migration ellipsoid, expressed as a parametric function of the angles $\alpha$ and $\beta$

$$
\begin{aligned}
\tau_{\xi} & =t_{N} \sin \alpha \cos \beta, \\
x_{\xi} & =\frac{t_{D} V}{2} \cos \alpha, \\
y_{\xi} & =\frac{t_{N} V}{2} \sin \alpha \sin \beta,
\end{aligned}
$$

where $t_{D}$ is the time of the input impulse and $t_{N}$ is the time after application of NMO. We differentiate the image coordinates with respect to the angles $\alpha$ and $\beta$; that is,

$$
\begin{aligned}
d \tau_{\xi} & =t_{N}(\cos \alpha \cos \beta d \alpha-\sin \alpha \sin \beta d \beta) \\
d x_{\xi} & =\frac{t_{D} V}{2}(-\sin \alpha d \alpha) \\
d y_{\xi} & =\frac{t_{N} V}{2}(\cos \alpha \sin \beta d \alpha+\sin \alpha \cos \beta d \beta) .
\end{aligned}
$$

We then eliminate the differentials $d \alpha$ and $d \beta$ from this set of equations by setting respectively $d y_{\xi}$ equal to zero when evaluating the $\operatorname{dip} p_{x}^{\xi}$ in the in-line direction, and set $d x_{\xi}$ equal to zero when evaluating the dip in the cross-line direction $p_{y}^{\xi}$. The second step is to eliminate the angles themselves and express the image dips as a function of the image coordinates $\left(\tau_{\xi}, x_{\xi}, y_{\xi}\right)$,

$$
\begin{aligned}
& p_{x}^{\xi}=\frac{d \tau_{\xi}}{d x_{\xi}}=\frac{2 t_{N}}{V t_{D}} \frac{\cot \alpha}{\cos \beta}=\frac{4 t_{N}^{2} x_{\xi}}{V^{2} t_{D}^{2} \tau_{\xi}} \\
& p_{y}^{\xi}=\frac{d \tau_{\xi}}{d y_{\xi}}=\frac{2 \tan \beta}{V}=\frac{4 y_{\xi}}{V^{2} \tau_{\xi}} .
\end{aligned}
$$

The wavelet-stretch factor can be easily derived by differentiating the summation surfaces of 3-D prestack time migration expressed as the hyperboloids

$$
t_{D}=t_{s}+t_{g}=\sqrt{\frac{\tau_{\xi}^{2}}{4}+\frac{\left|\vec{\xi}_{\mathbf{x y}}-\overrightarrow{\mathbf{s}}\right|^{2}}{V^{2}}}+\sqrt{\frac{\tau_{\xi}^{2}}{4}+\frac{\left|\vec{\xi}_{\mathbf{x y}}-\overrightarrow{\mathbf{g}}\right|^{2}}{V^{2}}}
$$


where $\overrightarrow{\mathbf{s}}$ and $\overrightarrow{\mathrm{g}}$ are the source and receiver coordinates vector, and $\vec{\xi}_{\mathbf{x y}}=\left(x_{\xi}, y_{\xi}\right)$ represents the horizontal components of the image coordinates vector. After a few simple algebra steps, we obtain

$$
\frac{d t_{D}}{d \tau_{\xi}}=\frac{\tau_{\xi}}{4}\left(\frac{1}{t_{s}}+\frac{1}{t_{g}}\right) .
$$

